Indirect measurement of poloidal rotation using inboard-outboard asymmetry of toroidal rotation and comparison with neoclassical predictions

This article has been downloaded from IOPscience. Please scroll down to see the full text article.

2013 Nucl. Fusion 53023002

(http://iopscience.iop.org/0029-5515/53/2/023002)

View the table of contents for this issue, or go to the journal homepage for more

Download details:

IP Address: 128.178.125.176

The article was downloaded on 18/01/2013 at $15: 18$

Please note that terms and conditions apply. 


\title{
Indirect measurement of poloidal rotation using inboard-outboard asymmetry of toroidal rotation and comparison with neoclassical predictions
}

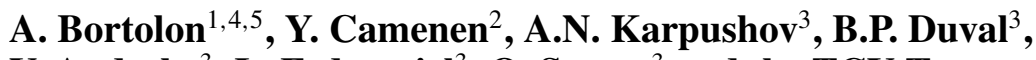 \\ Y. Andrebe ${ }^{3}$, L. Federspiel ${ }^{3}$, O. Sauter ${ }^{3}$ and the TCV Team \\ ${ }^{1}$ Department of Physics and Astronomy, University of California, Irvine, Irvine, CA 92697, \\ USA \\ 2 Aix-Marseille Université, CNRS, PIIM UMR 7345, Marseille, France \\ ${ }^{3}$ Ecole Polytechnique Fédérale de Lausanne (EPFL), Centre de Recherches en Physique des \\ Plasmas, Association Euratom-Confédération Suisse, CH-1015 Lausanne, Switzerland \\ E-mail: abortolo@pppl.gov
}

Received 2 September 2012, accepted for publication 12 December 2012

Published 15 January 2013

Online at stacks.iop.org/NF/53/023002

\begin{abstract}
An alternative experimental spectroscopic measurement of poloidal plasma rotation in toroidally confined plasmas is proven effective in the TCV tokamak. Charge exchange recombination measurements of the toroidal rotation profile over the full mid-plane plasma diameter are used to infer the complete bi-dimensional flow structure of the intrinsic $C^{6+}$ impurity, which includes its poloidal component. For divergence free flows, the difference between the toroidal rotation frequency $f_{\mathrm{t}}=u_{\mathrm{t}} / R$ at the inboard and outboard locations on the same flux surface is proportional to the poloidal rotation. This indirect measurement provides increased accuracy as the measured quantity $f_{\mathrm{t}, \text { in }}-f_{\mathrm{t} \text {,out }} \approx 4 q u_{\mathrm{p}} / R_{\text {axis }}$ ( $q$ is the local safety factor) is larger than the intrinsic uncertainties of a direct spectroscopic measurement of poloidal velocity. The method is applied in a variety of TCV ohmic and electron cyclotron heated L-mode plasmas in the banana-plateau collisionality regime $\left(0.2<v_{\mathrm{ii}}^{*}<2.4\right)$. In the radial range of normalized poloidal flux $\rho_{\psi}<0.8$, an impurity poloidal velocity of $u_{\mathrm{p}}=0.5-2.5 \mathrm{~km} \mathrm{~s}^{-1}$ is observed, always in the electron diamagnetic drift direction. The measurements are compared with neoclassical calculations and they agree in magnitude and sign to within $<1 \mathrm{~km} \mathrm{~s}^{-1}$.
\end{abstract}

(Some figures may appear in colour only in the online journal)

\section{Introduction}

Poloidal plasma rotation is thought to be a key player for many aspects of magnetically confined plasmas. It is, by example, predicted to induce transitions in magnetohydrodynamic (MHD) equilibrium [1,2] and to affect the stability of different types of MHD modes [3-6]. More generally, poloidal rotation enters the radial force balance equation, which is used to determine experimentally the radial electric field, an essential element in the physics of microinstability and turbulent transport [7]. Accurate measurements and predictions of

\footnotetext{
4 Present address: Department of Nuclear Engineering, University of Tennessee, Knoxville, TN, USA.

${ }_{5}^{5}$ Present address: Princeton Plasma Physics Laboratory, PO Box 451, MS34,
} Princeton, NJ 08543-0451, USA. poloidal velocity are, thus, highly desirable in present and future tokamak devices.

The majority of velocity measurements are obtained using spectroscopic diagnostics, which measure the Doppler shift of known emission lines. At present, velocities of the order of $10-100 \mathrm{~km} \mathrm{~s}^{-1}$ may be accurately measured but, in most experiments, the poloidal rotation is typically predicted and observed to be a few $\mathrm{km} \mathrm{s}^{-1}$. The associated Doppler shift is only a small fraction of the thermal line broadening and of the order of the best measurement accuracy available, $\approx 1 \mathrm{~km} \mathrm{~s}^{-1}$ [8].

Measurements at this level are further complicated by systematic issues. For diagnostics exploiting passive line emission, the integration of the emissivity along the line of sight may easily dominate the measured line shapes. Abel 
inversion techniques can, in principle, be used to localize the measurement [9], but usually at the cost of larger uncertainties.

Diagnostics relying on active charge exchange (CX) recombination $[10,11]$ have the benefit of localized measurements, but, for instance, the energy dependence of the CX cross sections and the dependence of the rotationfree wavelength on plasma parameters can give rise to apparent velocity contributions that are difficult to compensate even with extensive modelling and/or costly diagnostic configurations $[8,12]$.

For many cases of interest, direct measurements often struggle to attain the accuracy required to obtain experimental conclusions about the poloidal rotation amplitude or even direction, inhibiting comparison with theoretical predictions (e.g. from neoclassical or turbulent models).

Despite these difficulties, the experimental study of poloidal rotation and its comparison with the neoclassical theory remains an active field of research. Poloidal velocities of light impurities $(\mathrm{C}, \mathrm{O})$ have been reported that are consistent with neoclassical predictions [8,13-17]. In often more extreme situations, others note departures from neoclassical predictions [18-20] and the search for mechanisms able to drive poloidal rotation beyond the limit set by neoclassical damping remains actively debated.

This paper reports a novel measurement technique to demonstrate that the structure of plasma flows on a flux surface can be exploited effectively to obtain an indirect measurement of poloidal rotation, with an accuracy sufficient to test neoclassical predictions to the order of $2 \mathrm{~km} \mathrm{~s}^{-1}$.

For toroidally confined plasmas, characterized by incompressible flows, the toroidal rotation frequency $f_{\mathrm{t}}=$ $u_{\mathrm{t}} / R$ varies on a flux surface by an amount proportional to the poloidal rotation $\left(u_{\mathrm{t}}\right.$ is the toroidal velocity and $R$ is the major radius). From the difference between the toroidal frequency measured at the outboard (low-field side, L subscript) and inboard (high-field side, $\mathrm{H}$ subscript) sides of the same flux surface $\Delta f_{\mathrm{t}}=f_{\mathrm{t}, \mathrm{H}}-f_{\mathrm{t}, \mathrm{L}}$ an indirect measurement of poloidal velocity $u_{\mathrm{p}}$ can be obtained. In the large aspect-ratio limit this difference scales favourably with the safety factor $q$ :

$$
\Delta f_{\mathrm{t}} \approx \frac{4 q}{R_{0}} u_{\mathrm{p}}
$$

where $R_{0}$ is the radial location of the magnetic axis and $u_{\mathrm{p}}$ is the poloidal rotation at the LFS. Poloidal velocities of $\mathrm{km} \mathrm{s}^{-1}$ generate inboard-outboard asymmetries of toroidal frequency that can easily become larger than the systematic uncertainties inherent in the direct measurement of $u_{\mathrm{p}}$ from Doppler shift spectroscopic lines.

The indirect measurement method, recently outlined in [21], was critically tested and then extensively used to measure the poloidal rotation in the tokamak TCV [22], where $\mathrm{CX}$ measurements of toroidal carbon rotation are available with full minor-diameter coverage. The accuracy achieved allowed the comparison with the neoclassical theory for a wide range of low confinement plasma scenarios. The rest of the paper is organized as follows: in section 2 after establishing the theoretical background, the principle of the indirect measurement is presented and its domain of applicability discussed. The experimental configuration, diagnostic configuration and plasma characteristics are described in section 3. Examples of indirect poloidal rotation measurements are presented in detail in section 4 . In section 5 the results of indirect measurements on a large dataset of plasma discharges are compared with the predictions from the neoclassical theory. The concluding section summarizes the results and discusses benefits and issues associated with the indirect measurement of poloidal rotation.

\section{Theoretical background and measurement principle}

In this section, the indirect poloidal rotation measurement method and its applicability conditions are discussed. The presented material recalls the essential results of the neoclassical theory with more details available in the literature (e.g. $[23,24])$. Some of the formulae depend explicitly on the conventions adopted for the coordinate system. General expressions applicable in any convention system are provided in appendix $\mathrm{B}$, based on the COCOS formalism introduced in [25].

In the following derivation, a right-handed coordinate system is taken $(\psi, \theta, \varphi)$ with $\psi$ the poloidal magnetic flux (minimum on axis), $\theta$ a poloidal angle (zero at LFS mid-plane, increasing towards the top of the torus) and $\varphi$ the toroidal angle (clockwise when viewed from above).

The magnetic field may be written as

$$
\boldsymbol{B}=s_{b} F \nabla \varphi+s_{j} \nabla \varphi \times \nabla \psi
$$

where $s_{b}$ and $s_{j}$ indicate the direction of the magnetic field and plasma current with respect to the toroidal direction $\nabla \varphi$ and $F=R B_{\mathrm{t}}>0$ with $B_{\mathrm{t}}$ the toroidal magnetic field.

To first order in $\rho_{\mathrm{p}}^{*}$ (the poloidal Larmor radius normalized to the perpendicular scale length) the fluid velocity of the considered species is

$$
\boldsymbol{u}=u_{\|} \boldsymbol{b}+\frac{\boldsymbol{b} \times \nabla \boldsymbol{\phi}}{B}+\frac{\boldsymbol{b} \times \nabla p}{n Z e B},
$$

where $u_{\|}$is the component of the velocity along the magnetic field, $\boldsymbol{b}=\boldsymbol{B} /|\boldsymbol{B}|, \phi$ is the electrostatic potential, $e$ is the electron charge and $n, p, Z$ are, respectively, the species density, pressure and charge number. The second and third terms on the right-hand side correspond to the perpendicular velocity, separated into electrostatic and diamagnetic components. Algebraic manipulations with the use of the relation

$$
R^{2} \nabla \varphi=s_{b} F \frac{B}{B^{2}}+s_{j} \frac{\nabla \psi \times B}{B^{2}},
$$

allow the expression of the flow of the selected species in the form

$$
\boldsymbol{u}=\hat{u} \boldsymbol{B}+\hat{\omega} \boldsymbol{R e}_{\varphi},
$$

where $e_{\varphi}=R \nabla \varphi$ and

$$
\begin{aligned}
\hat{u}(\psi, \theta) & =\frac{\boldsymbol{u} \cdot \nabla \theta}{\boldsymbol{B} \cdot \nabla \theta}, \\
\hat{\omega}(\psi, \theta) & =-s_{j}\left[\frac{\partial \phi}{\partial \psi}+\frac{1}{Z e n} \frac{\partial p}{\partial \psi}\right] .
\end{aligned}
$$

Note that, at this stage, $\hat{u}$ and $\hat{\omega}$ retain a possible dependence on the poloidal angle. The continuity and momentum balance 
equations will put additional constraints on the first-order flow. The continuity equation may be written as

$$
\frac{\partial n}{\partial t}+\nabla \cdot(n \boldsymbol{u})=\mathcal{S}_{n}
$$

where $\boldsymbol{u}$ and $\mathcal{S}_{n}$ are the fluid velocity and the particle source of the considered species, respectively. Assuming axisymmetry, the divergence of the particle flux is

$$
\nabla \cdot(n \boldsymbol{u})=B^{\theta}\left[\frac{\partial}{\partial \psi}\left(\frac{n u^{\psi}}{B^{\theta}}\right)+\frac{\partial}{\partial \theta}\left(\frac{n u^{\theta}}{B^{\theta}}\right)\right],
$$

where the upperscript indicates the contravariant component (i.e. $u^{\theta}=\boldsymbol{u} \cdot \nabla \theta$ ). Inserting this expression into equation (8) and integrating over the poloidal angle yields

$n \hat{u}(\theta)-n \hat{u}(0)=\int_{0}^{\theta} \frac{\mathrm{d} \theta}{B^{\theta}}\left[\mathcal{S}_{n}-\frac{\partial n}{\partial t}-B^{\theta} \frac{\partial}{\partial \psi}\left(\frac{n u^{\psi}}{B^{\theta}}\right)\right]$.

Assuming a stationary density, no particle source (for the considered species) and a negligible radial derivative of the radial particle flux at all poloidal angles, the quantity $n \hat{u}$ is constant over a flux surface so

$$
\hat{u}(\psi, \theta)=\frac{K(\psi)}{n(\psi, \theta)} .
$$

If the density is not a flux function, $\hat{u}$ depends on the poloidal angle. This may indeed occur in strongly rotating plasmas (or even at moderate rotation for heavy impurities) where the centrifugal force induces poloidal asymmetries in the density and electrostatic potential [26]. At the plasma edge, the poloidal variation of the parallel friction between the bulk ions and impurities may also engender density asymmetries [27, 28].

In the plasmas considered in this study, the centrifugal effect on carbon impurities remains modest $m_{\mathrm{C}} u_{\mathrm{C}}^{2} /\left(2 T_{\mathrm{C}}\right)<$ 0.25 with a negligible predicted density asymmetry between HFS and LFS for carbon $\left(0.96<n_{\mathrm{C}, \mathrm{H}} / n_{\mathrm{C}, \mathrm{L}}<1\right.$ [26]) and an even weaker effect for deuterium. The effect of inertial forces on the electrostatic potential and poloidal density asymmetries of both species will thus be neglected (note: this does not necessarily imply that the impact of inertial forces on turbulent transport can be neglected). Furthermore, the plasma collisionality is sufficiently low that the effect of parallel friction on the impurities may also be neglected. The poloidal modulation of impurity density due to parallel friction can be assessed by equating the impurity pressure gradient and friction in the parallel force balance. The poloidal impurity density modulation is then found to be characterized by the parameter $\Delta$ defined in equation (8) of [27] which quantifies the ability of the friction force to drive a parallel density gradient. In the plasmas considered here, $\Delta$ is less than $2 \%$ for carbon.

The plasma density and (first order) electrostatic potential are therefore taken to be flux functions. Here, the first-order fluid velocity of each plasma species lies entirely on flux surfaces and can be expressed as the sum of a toroidal rigidbody component and a component parallel to the magnetic field [24], fully specified by flux constant quantities $\hat{\omega}(\psi)$ and $\hat{u}(\psi)$ :

$$
\boldsymbol{u}=\hat{\omega}(\psi) \boldsymbol{R e}_{\varphi}+\hat{u}(\psi) \boldsymbol{B} .
$$

Equation (12), projected onto toroidal (along $\nabla \varphi$ ) and poloidal (along $\nabla \varphi \times \nabla \psi$ ) direction, may be written as

$$
\begin{aligned}
& u_{\mathrm{t}}=\hat{\omega}(\psi) R+s_{b} \hat{u}(\psi) F(\psi) / R, \\
& u_{\mathrm{p}}=s_{j} \hat{u}(\psi) B_{\mathrm{p}}
\end{aligned}
$$

with $B_{\mathrm{p}}=|\nabla \varphi \times \nabla \psi|$ the norm of the poloidal magnetic field. In the absence of poloidal rotation $u_{\mathrm{p}}=\hat{u}(\psi)=0$ and each plasma surface rotates toroidally as a rigid body at a rotation frequency $f_{\mathrm{t}}=u_{\mathrm{t}} / R=\hat{\omega}(\psi)$. Conversely, a finite $u_{\mathrm{p}}$ is associated with a contribution $\hat{u}(\psi) F(\psi) / R^{2}$ to the local toroidal frequency that varies on the flux surface. A measurement of the difference $\Delta f_{\mathrm{t}}=f_{\mathrm{t}, \mathrm{H}}-f_{\mathrm{t}, \mathrm{L}} \propto \hat{u}(\psi)$ is thus equivalent to a measurement of $u_{\mathrm{p}}$.

By evaluating equation (13) at the HFS and LFS midplane, explicit expressions for the flux functions $\hat{\omega}(\psi)$ and $\hat{u}(\psi)$ are obtained:

$$
\begin{aligned}
& \hat{\omega}(\psi)=\left(u_{\mathrm{t}, \mathrm{H}} R_{\mathrm{H}}-u_{\mathrm{t}, \mathrm{L}} R_{\mathrm{L}}\right) \times\left(R_{\mathrm{H}}^{2}-R_{\mathrm{L}}^{2}\right)^{-1}, \\
& \hat{u}(\psi)=\frac{s_{b}}{F}\left(\frac{u_{\mathrm{t}, \mathrm{H}}}{R_{\mathrm{H}}}-\frac{u_{\mathrm{t}, \mathrm{L}}}{R_{\mathrm{L}}}\right) \times\left(\frac{1}{R_{\mathrm{H}}^{2}}-\frac{1}{R_{\mathrm{L}}^{2}}\right)^{-1} .
\end{aligned}
$$

Thus, provided the magnetic equilibrium is known to the required accuracy, a combined measurement of HFS and LFS toroidal rotation profiles is sufficient to deduce completely the bi-dimensional pattern of toroidal and poloidal flows. Moreover, evaluating equation (7) along the plasma mid-plane, provides an expression for the radial electric field showing that it is determined only by the rigid body portion of the toroidal rotation (combining equation (13) with the radial force balance equation leads to the same result):

$$
E_{\mathrm{R}}=\frac{1}{n Z e} \frac{\partial p}{\partial R}+s_{j} \hat{\omega}(\psi) R B_{\mathrm{p}} .
$$

Since the quantity $\hat{\omega}(\psi)$ can be obtained experimentally from equation (15), from a measurement of the inboard-outboard asymmetry of $f_{\mathrm{t}}$ one can, in principle, accurately deduce the radial electric field, using only toroidal viewed measurements.

If the electrostatic potential is a flux function, equations (5), (6) and (7) can be simply combined to obtain a variant of equation (16) accounting for arbitrary poloidal density variations. This would be the case if the impurity concentration is small and the inertial effects are negligible for the main ions. The electrostatic potential is then still expected to be constant on a flux surface, as is the main ion density (from the lowest order parallel force balance $\boldsymbol{b} \cdot \nabla p_{\mathrm{i}}=-e n_{\mathrm{i}} \boldsymbol{b} \cdot \nabla \boldsymbol{\phi}$, where $p_{\mathrm{i}}$ the main ion pressure). For the impurities, assuming that the temperature is a flux function, equation (16) becomes

$$
\begin{aligned}
K(\psi)= & \frac{s_{b}}{F}\left(\frac{u_{\mathrm{t}, \mathrm{H}}}{R_{\mathrm{H}}}-\frac{u_{\mathrm{t}, \mathrm{L}}}{R_{\mathrm{L}}}-s_{j} \frac{T}{Z e} \frac{\partial \ln n_{\mathrm{H}} / n_{\mathrm{L}}}{\partial \psi}\right) \\
& \times\left(\frac{1}{n_{\mathrm{H}} R_{\mathrm{H}}^{2}}-\frac{1}{n_{\mathrm{L}} R_{\mathrm{L}}^{2}}\right)^{-1}
\end{aligned}
$$

with $n \hat{u}=K(\psi)$ and $n_{\mathrm{H}}, n_{\mathrm{L}}$ the impurity density at the highand low-field sides, respectively. The origin of any impurity asymmetry is left unspecified. The above equation will be used in section 4 to test the impact of observed carbon density asymmetries on the indirect poloidal rotation measurement.

In this section, we have showed that, under rather general conditions, the asymmetry of toroidal frequency can be 
interpreted as an indirect measurement of poloidal velocity $u_{\mathrm{p}}$. From an experimental perspective, the indirect measurement has also an interesting potential for improved precision and accuracy. This is qualitatively understood from the $4 q$ amplification factor in equation (1). To address the question quantitatively, we rewrite equation (1) to express the poloidal rotation as a function of the toroidal rotation measured at LFS and HFS locations ( $\mathrm{L}$ and $\mathrm{H}$ subscripts respectively) of the same flux surface:

$$
u_{\mathrm{p}} \approx \frac{1}{4 q}\left(\Delta u_{\mathrm{t}}+2 \epsilon \bar{u}_{\mathrm{t}}\right),
$$

where $\Delta u_{\mathrm{t}}=u_{\mathrm{t}, \mathrm{H}}-u_{\mathrm{t}, \mathrm{L}}, \bar{u}_{\mathrm{t}}=\left(u_{\mathrm{t}, \mathrm{H}}-u_{\mathrm{t}, \mathrm{L}}\right) / 2, \epsilon=r / R_{0}$ is the local inverse aspect ratio and $u_{\mathrm{p}}$ the poloidal rotation at the LFS. The term $2 \epsilon \bar{u}_{\mathrm{t}}$ at the right-hand side represents the part of toroidal velocity asymmetry associated with toroidicity $\left(R_{\mathrm{H}}<R_{\mathrm{L}}\right)$.

If the measurements $u_{\mathrm{t}, \mathrm{H}}$, and $u_{\mathrm{t}, \mathrm{L}}$ are affected by random errors $\sigma_{\mathrm{t}, \mathrm{L}} \approx \sigma_{\mathrm{t}, \mathrm{H}} \approx \sigma_{\mathrm{t}}$, this uncertainty will propagate to a poloidal velocity error $\sigma_{\mathrm{p}} \approx \sigma_{\mathrm{t}} /(q \sqrt{2})$. At the same time, systematic errors $\delta u_{\mathrm{t}, \mathrm{L}} \approx \delta u_{\mathrm{t}, \mathrm{H}} \approx \delta u_{\mathrm{t}}$, for example those associated with the wavelength calibration or the value of the wavelength at rest, will partially cancel algebraically in equation (19), and, as a result, the indirect measurement of poloidal rotation will be affected by an off-set error $\delta u_{\mathrm{p}} \approx$ $\delta u_{\mathrm{t}} \epsilon /(2 q)$. For magnetic configurations typical of TCV, this factor is easily lower than 0.05 , making the indirect measurement extremely robust against off-set type of errors.

This error analysis only considers uncertainties associated with toroidal rotation measurements. Other error sources specific of the indirect measurement can degrade the accuracy and precision of the method, for example those descending from the approximate knowledge of the magnetic equilibrium. A detailed description of error analysis on experimental cases will be provided in section 4 .

\section{Experimental setup}

Although the link between poloidal rotation and toroidal rotation was recognized in early theoretical studies, (e.g. [29], where the importance of the asymmetrical part of toroidal frequency in the edge region was emphasized), measurement of rotation over the inboard region of a tokamak plasma is uncommon. This can be mostly ascribed to practical considerations such as limited diagnostic access to the inboard side, constrained by the presence of the central solenoid.

Active CX measurements require a neutral donor population, usually provided by heating or diagnostic beams; in many devices, these beams do not reach the inboard region, either as they are aligned tangentially or due to strong attenuation when traversing the plasma core.

Following a growing interest in the inboard plasma flow, the situation is progressively changing. Gas puff techniques from central column injectors are used for inboard $C X$ measurements, but the spatial coverage remains restricted to the extreme plasma periphery as the donor cloud penetrates only a short distance into the confined plasma region $[13,30]$. Alternatively, the neutral beam geometry and energy can be optimized for radial penetration, as on the TCV tokamak, where a diagnostic neutral beam (DNB) was used to obtain the first spatially and temporally resolved measurements of toroidal rotation across the full plasma diameter [31], and address the structure of first-order plasma flows. Recently, similar measurements were also reported from the DIII-D tokamak [21].

TCV is a medium sized tokamak $\left(R_{0}=0.88 \mathrm{~m}, a=\right.$ $\left.0.25 \mathrm{~m}, B_{\mathrm{t}}=1.45 \mathrm{~T}, I_{\mathrm{p}}<1 \mathrm{MA}\right)$, with an elongated vacuum vessel and a flexible control system that can generate a wide variety of plasma shapes [22]. Up to $4.5 \mathrm{MW}$ of auxiliary electron cyclotron heating $(\mathrm{ECH})$ is available from nine gyrotron sources. No auxiliary ion heating was used for the experiments reported herein. Plasma rotation is measured by the CXRS diagnostic [31-33] from the Doppler-shifted active CX emission of carbon impurities (optimized for CVI $(n=8 \rightarrow 7), 529.1 \mathrm{~nm})$.

The neutral donor population is provided by a quasiperpendicular diagnostic neutral beam (DNB) [34], which injects neutral hydrogen at an angle of $11.25^{\circ}$ in the counterclockwise toroidal direction as viewed from above. The beam acceleration voltage is $V_{\mathrm{DNB}}=50 \mathrm{kV}$. With a cold cathode arcdischarge plasma generator and tailored ion optical focusing, proton $\left(\mathrm{H}^{+}\right)$beam fractions of $85 \%$ and beam divergence of $0.5^{\circ}$ are attained, at an equivalent neutral current of $I_{\mathrm{DNB}} \approx$ 1.6 A. Typically operated with pulses with a $1: 2$ duty cycle for spectral background subtraction, the DNB $(<80 \mathrm{~kW}$ injected into tokamak) perturbation of energy and momentum balance may be neglected.

The CXRS diagnostic comprises three separate diagnostic systems, each constituted by an optical collection system consisting in a periscope and a focusing lens; a bundle of 40 optic fibres that transport the collected light to the measurement instruments; a monochromator that resolves the spectral features of the observed line; and a CCD camera working as a multi-channel detection element. Light collected from each system is analysed by a separate monochromator, equipped with double slit at the entrance and a demagnifying element at the exit allowing 40 spectra on the CCD detector to be imaged simultaneously. The collection systems are arranged to provide two horizontal views and one vertical view (figure 1). Each system collects light from two arrays of 20 lines of sight (LOS), aligned so as to sample the same radial locations along the DNB path, at the vessel mid-plane.

Overall, the HFS and LFS horizontal systems measure the toroidal rotation over the full plasma diameter with a slight overlap at the machine centre, with LOS spaced by $\approx 15 \mathrm{~mm}$ at the intersection with the beam. The vertical CXRS view used for direct poloidal rotation measurements has a smaller LOS spacing $\approx 7 \mathrm{~mm}$, but only covers the outer half of the outboard minor radius $\left(\rho_{\psi}>0.4\right)$. The diagnostic temporal resolution is determined by the detector exposure time which, for these experiments, varied between 12 and $30 \mathrm{~ms}$. A spectroscopic wavelength calibration is performed using reference spectra from a cold neon tube, automatically acquired on all spectrometers after each plasma discharge. It is important to note that, owing to the low ion temperatures $\left(T_{\mathrm{i}}<900 \mathrm{eV}\right)$ and the nearly perpendicular observation of the beam, the apparent velocity component associated with the energy dependence of the CX cross section may safely be neglected particularly as the full energy beam component dominates the CVI $(n=8 \rightarrow 7)$ spectral line intensity [31].

In the reported experiments, unless stated otherwise, deuterium plasmas were in the L-mode confinement regime, 

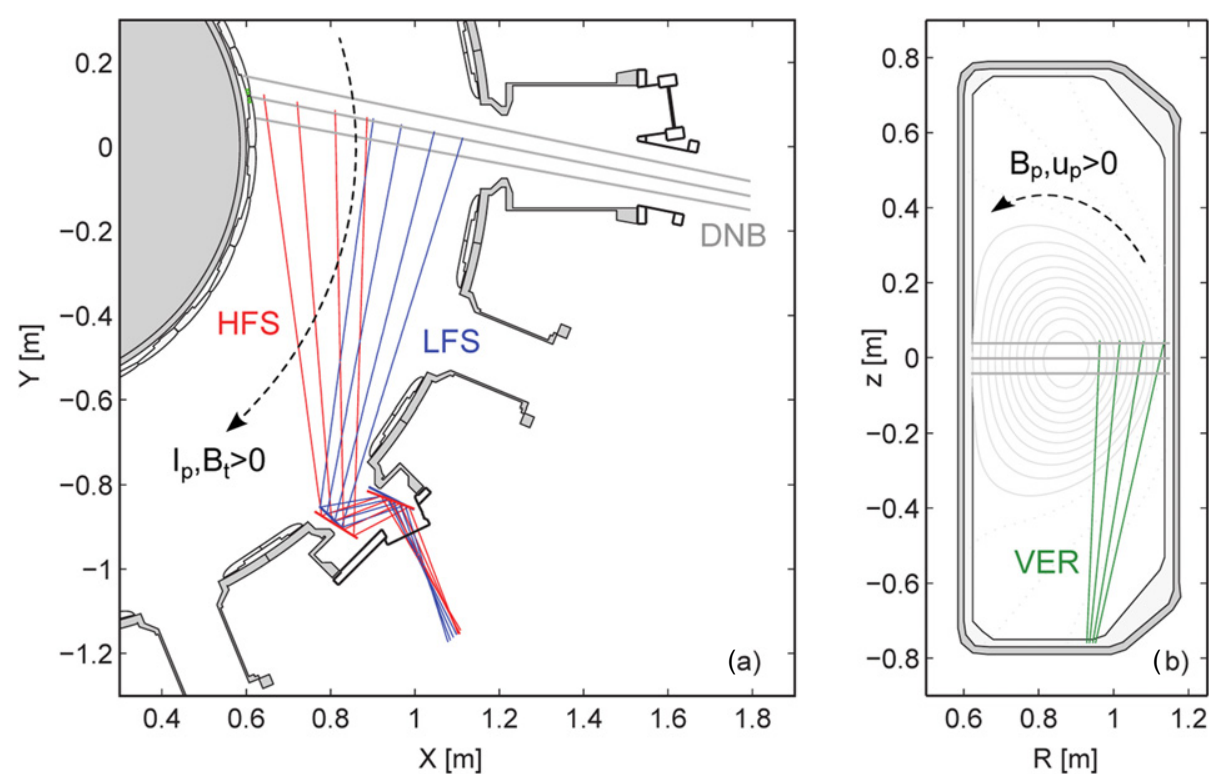

Figure 1. Horizontal $(a)$ and vertical $(b)$ sections of the TCV device. Schematic of the DNB footprint and the observation geometry of the CXRS diagnostic systems are shown (for each system, only 4 of the 40 chords are displayed). Sign conventions for the toroidally and poloidally directed quantities are indicated.

with the central column acting as limiter. The direction of $I_{\mathrm{p}}$ and $B_{\mathrm{t}}$ were changed independently. As in section $2, u_{\mathrm{t}}$, $I_{\mathrm{p}}$ and $B_{\mathrm{t}}$ are positive if clockwise from the top; $u_{\mathrm{p}}$ and $B_{\mathrm{p}}$ are positive if upward at the outboard mid-plane (figure 1) (opposite to usual TCV convention). The normalized poloidal flux $\rho_{\psi}=\sqrt{\left(\psi-\psi_{0}\right) /\left(\psi_{a}-\psi_{0}\right)}$ is used as the radial flux coordinate, with the subscript 0 indicating the magnetic axis and $a$ is the plasma boundary.

\section{Examples of indirect poloidal measurements}

A detailed analysis for TCV $45142\left(I_{\mathrm{p}}=+160 \mathrm{kA}, B_{\mathrm{t}}=\right.$ $+1.42 \mathrm{~T}$ ), where second harmonic ECH was used to modify the plasma rotation, is first presented and figure 2 shows the temporal evolution of selected plasma parameters. An initial ohmic phase is followed by two periods of increasing $\mathrm{ECH}$ from up to two gyrotrons, $P_{\mathrm{ECH}}=0.5 \mathrm{MW}$ at $0.75 \mathrm{~s}$ and $P_{\mathrm{ECH}}=1.0 \mathrm{MW}$ at $t=1.4 \mathrm{~s}$. The $\mathrm{ECH}$ power is deposited off-axis $\left(0.5<\rho_{\psi}<0.75\right)$, with $\approx 95 \%$ absorption. The plasma stored energy, measured by a diamagnetic loop, remains in the range $4-5.5 \mathrm{~kJ}$. During the $1.8 \mathrm{~s}$ duration flat top, the main plasma parameters such as plasma current $I_{\mathrm{p}}=$ $160 \mathrm{kA}$, electron density $n_{\mathrm{e}} \approx 3 \times 10^{19} \mathrm{~m}^{-3}, B_{\mathrm{t}}=1.4 \mathrm{~T}$ and edge safety factor $q_{\mathrm{e}}=7.5$ vary by $<5 \%$, permitting the analysis of extended time windows $(\sim 100 \mathrm{~ms})$ to increase the measurement statistics. During the ohmic phase, the plasma exhibits a sawtoothing instability with a small inversion radius $\rho_{\psi}=0.1$ and a period of $3 \mathrm{~ms}$, considerably shorter than the $12 \mathrm{~ms}$ exposure time of the CXRS diagnostic. The off-axis $\mathrm{ECH}$ then stabilizes the sawteeth after $t=0.75 \mathrm{~s}$.

At these intermediate densities $n_{\mathrm{e}} \approx 3 \times 10^{19} \mathrm{~m}^{-3}$, the full energy beam current density at the HFS edge, integrated along observation chords, is about $60 \%$ of that at the LFS, due to beam divergence and plasma attenuation. Figure $2(d)$ illustrates the intensity of the CX carbon line for two channels at $R=0.76 \mathrm{~m}$ and $R=1.00 \mathrm{~m}$, which lie on the same normalized poloidal flux surface $\rho_{\psi}=0.6$. The small intensity increases coincident with the DNB pulses, demonstrate the relatively low active to passive ratio of $A / P \sim 5-10 \%$. Figures $2(f)$ and $(g)$ show, for the same channels, the carbon spectra after subtraction of background emission, averaged over the time interval $0.85<t<1.35 \mathrm{~s}$ (first ECH phase). Typical values of $S / N \sim 5-10$ allow statistic uncertainties in the deduced rotation of the order of $5 \mathrm{~km} \mathrm{~s}^{-1}$ where poor photon statistics is the main source of uncertainty. CXRS intensities depend linearly on the carbon content and, with such a weak beam, the performance depends strongly on the background emission level. The latter can change substantially throughout the experimental campaign following the quality of wall conditioning.

The toroidal rotation velocity for the two selected channels is plotted in figure 2(e). Error bars include uncertainties associated with the spectral fit, wavelength calibration and background subtraction. In the ohmic phase, the plasma rotates in the counter-current direction (negative values) at both locations, with values of 25 and $10 \mathrm{~km} \mathrm{~s}^{-1}$. As the ECH input is increased, the rotation at the LFS and HFS increases towards positive values with an approximately constant offset of $\approx 10 \pm 2 \mathrm{~km} \mathrm{~s}^{-1}$.

Figure 3 shows the toroidal rotation velocity and rotation frequency, measured over ohmic $(t=0.55-0.74 \mathrm{~s})$ and $1 \mathrm{MW}$ $\mathrm{ECH}(t=1.51-1.76 \mathrm{~s})$ time windows. The figure combines measurements from the two diagnostic spectroscopic systems, for the HFS (red) and LFS (blue). As expected for this low density ohmic L-mode discharge [35-37], the plasma rotates in the counter-current direction $\left(u_{\mathrm{t}}<0\right)$ with central velocities $\approx 30 \mathrm{~km} \mathrm{~s}^{-1}$, smaller towards the edge. The flattening of the central region $R=0.80-0.93 \mathrm{~m}$ is associated with the periodic sawtooth relaxations. Within the uncertainties, the profiles match at the magnetic axis $\left(R_{\text {axis }}=0.88 \mathrm{~m}\right)$, confirming the relative wavelength calibration. Towards the plasma edge a strong asymmetry of $u_{\mathrm{t}}$ develops: the HFS profile 

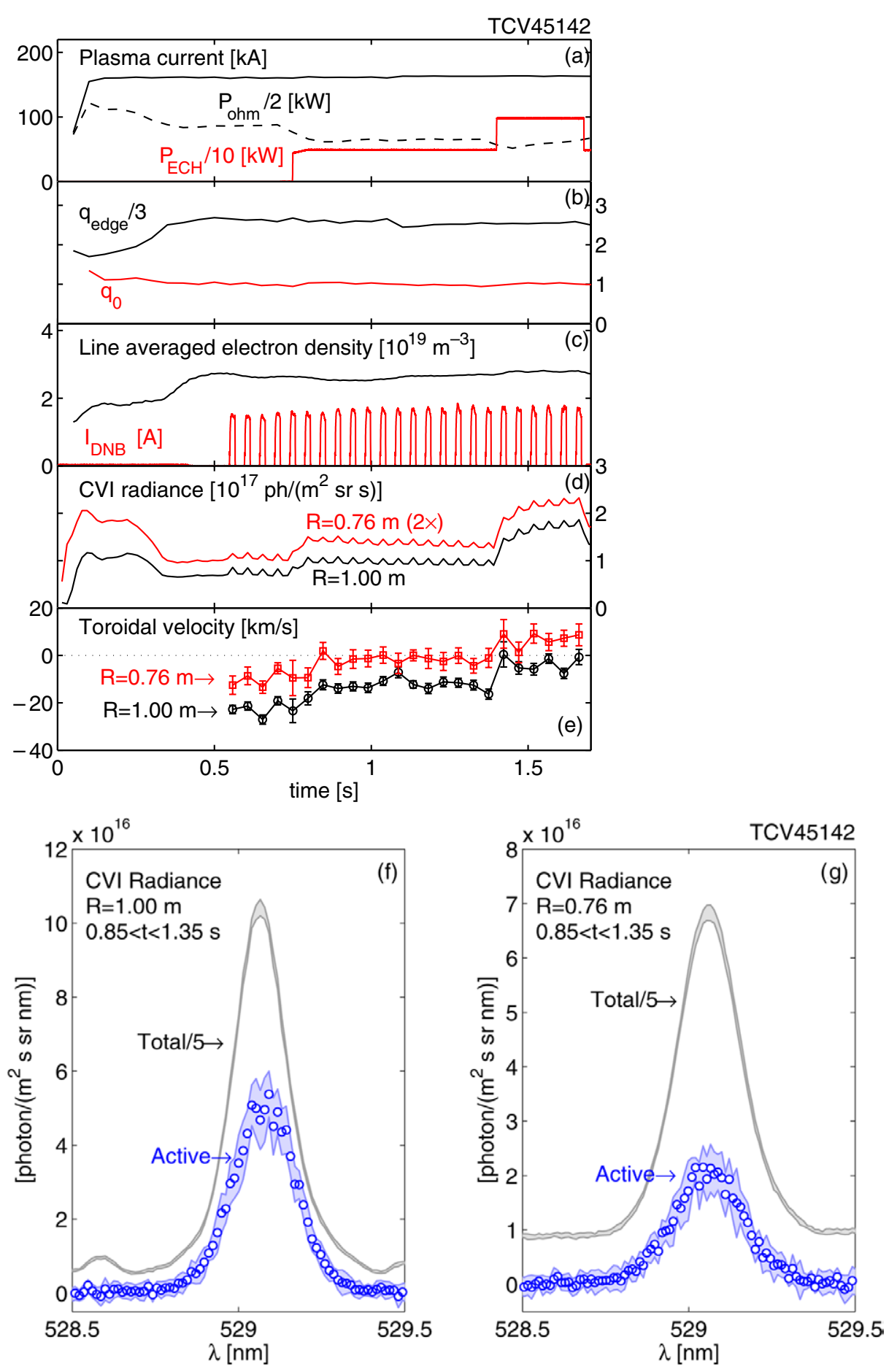

Figure 2. Evolution of discharge parameters for TCV discharge 45142: (a) plasma current $I_{\mathrm{p}}$, ohmic and ECH power $P_{\mathrm{ohm}}, P_{\mathrm{ECH}} ;(b)$ on axis and edge safety factor; $(c)$ electron density and DNB neutral equivalent current; $(d) C X$ intensity for two selected CXRS toroidal chords; $(e)$ toroidal rotation obseved by the same chords. CXRS spectra for two channels observing the same poloidal flux surface for the inboard $(f)$ and outboard $(g)$ regions. The spectra are averaged over the first ECH phase, $0.8<t<1.35 \mathrm{~s}$.

shows a steeper radial gradient so that for $R<0.73 \mathrm{~m}, u_{\mathrm{t}}$ becomes positive, indicative of reversed rotation direction (cocurrent). In the ECH phase, for the lower core velocity, this transition occurs further into the plasma at $R=0.78 \mathrm{~m}$ (figures 3(c) and $(d)$ ).

In figures $3(b)$ and $(d)$, the profiles of $f_{\mathrm{t}}$ are mapped to the flux label $\rho_{\psi}$, showing that the difference of HFS and LFS rotation increases radially, reaching values of $\Delta f_{\mathrm{t}} \approx 15 \mathrm{kHz}$ at $\rho_{\psi} \approx 0.85$. Interestingly, on some flux surfaces, the plasma rotates toroidally in the opposite direction at the HFS and LFS. This is clearly not compatible with a rigid body rotation but can be explained if the poloidal rotation is finite. In fact, the toroidal frequency asymmetries are consistent with a poloidal flow in the electron diamagnetic direction, generating a finite $\hat{u}(\psi) B_{\mathrm{t}} \sim 10 \mathrm{~km} \mathrm{~s}^{-1}$ contribution to the toroidal flow, in the co-current direction. 

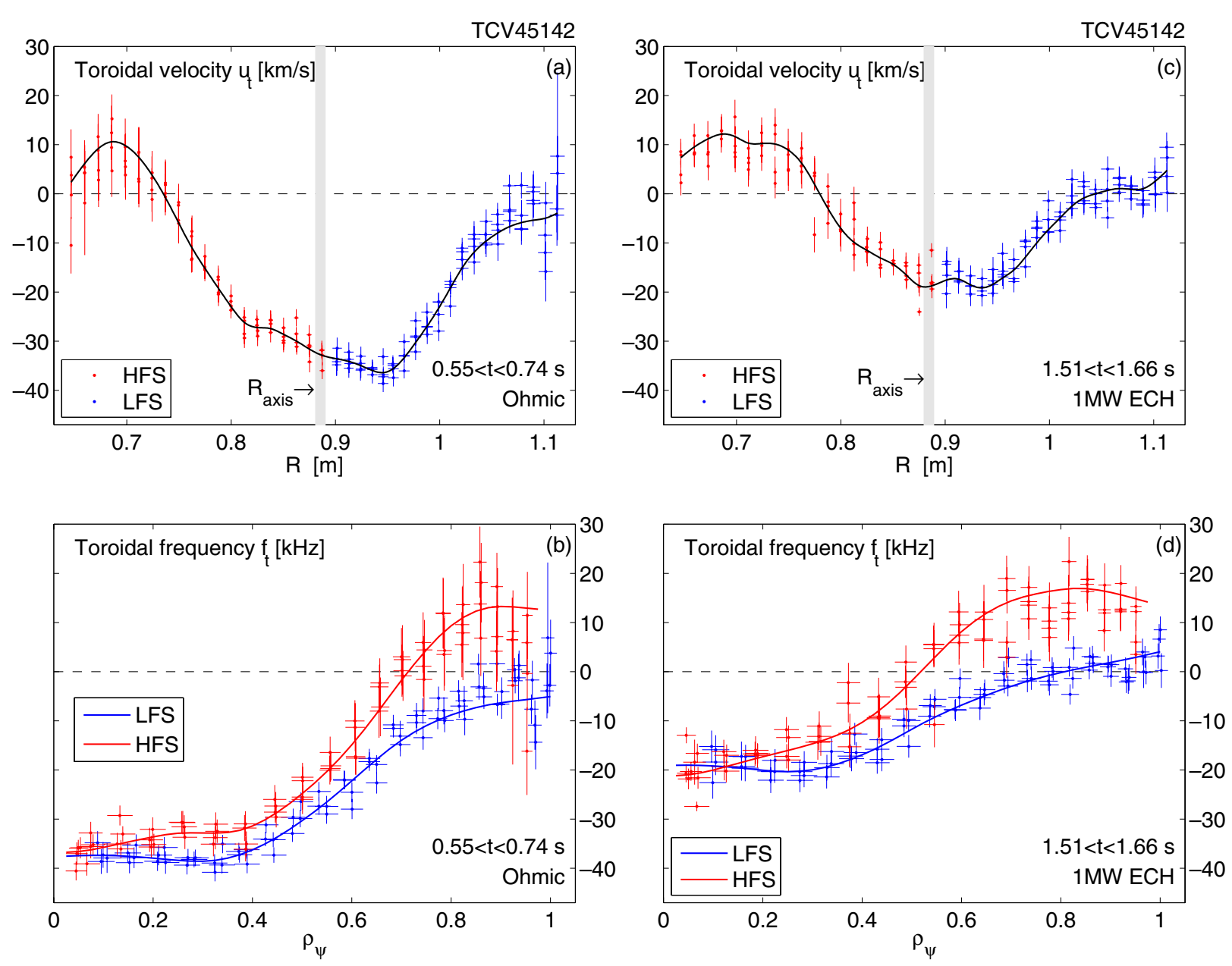

Figure 3. Profiles of toroidal rotation velocity $u_{\mathrm{t}}$ measured during ohmic $(a)$ and $\mathrm{ECH}(c)$ phases of TCV 45142 plasma discharge, with the corresponding toroidal rotation frequency $f_{\mathrm{t}}=u_{\mathrm{t}} / R$, at the HFS and LFS, plotted as a function of the flux label $\rho_{\psi}(b),(d)$.

The HFS and LFS measurements are now used to calculate the flux functions $\hat{\omega}$ and $\hat{u}$ that fully characterize the plasma flow through equation (5). First, the HFS and LFS profiles of $u_{\mathrm{t}}(\psi)$ are separately fitted with cubic spline curves as shown in figure 3; then equations (15) and (16) are used to compute $\hat{\omega}$ and $\hat{u}(\psi)$ from the fit curves. The results obtained for the ohmic phase are illustrated in figure $4(a)$. The rigid body component of the flow $\hat{\omega}$ is negative with a central frequency peaking at $-60 \mathrm{kHz}$ and the edge frequency around $-20 \mathrm{kHz}$. The flux function $\hat{u}$ is almost constant across the minor radius and about $10 \mathrm{~km} \mathrm{~s}^{-1} \mathrm{~T}^{-1}$. This implies that the total toroidal rotation frequency $u_{\mathrm{t}} / R$ is always more positive than its rigid body component $\hat{\omega}$, as can be checked by comparing with the $u_{\mathrm{t}} / R$ profiles at HFS and LFS of figure 3 . Once $\hat{u}(\psi)$ is known, it is easy to calculate the poloidal rotation at any location using $u_{\mathrm{p}}=s_{j} \hat{u}(\psi) B_{\mathrm{p}}$. The dependence of $u_{\mathrm{p}}$ on the poloidal angle is shown in figure $4(b)$ for three radial locations and simply reflects the poloidal variation of $B_{\mathrm{p}}$. As $B_{\mathrm{p}}$ decreases to zero towards the magnetic axis while $\hat{u}$ does not vary much, $u_{\mathrm{p}}$ is found decreasing with decreasing $\rho_{\psi}$.

The calculation of $\hat{\omega}$ and $\hat{u}$ depends critically on the accurate knowledge of the mapping functions $R_{\mathrm{H}}(\psi)$ and $R_{\mathrm{L}}(\psi)$ and the magnetic field components. To examine the accuracy of the equilibrium reconstruction the ion temperature profile $T_{\mathrm{i}}(\psi)$ obtained from the HFS and LFS systems are compared in figure $5(a)$. The profiles overlap within the measurement uncertainty, supporting the equilibrium reconstruction and the assumption that $T_{\mathrm{c}}$ is constant on a flux surface. The carbon density also appears to be in-out symmetric although there is some indication, at the limit of the measurement accuracy, of higher density at the LFS. In our analysis, we consider these quantities as poloidal flux functions and expect the plasma flows to be structured as described in equation (5). The impact of density asymmetries on the measurement will be assessed a posteriori in section 5 .

Uncertainties in the indirect $u_{\mathrm{p}}$ estimation arise from uncertainties in the Doppler-shift rotation measurements, the magnetic reconstruction mapping and the local magnetic field value. The total uncertainty of the $u_{\mathrm{p}}$ profile is determined using a Monte Carlo method, described in detail in appendix A. A statistically relevant ensemble of $M=1000$ of $u_{\mathrm{p}}$ profiles is computed from the experimental input profiles of $u_{\mathrm{t}}(R)$ and $B_{\mathrm{t}}(R)$, accounting for the uncertainties of the rotation values, the measurement locations and the equilibrium reconstruction. To estimate the uncertainties in the $R_{\mathrm{H}}(\psi)$ and $R_{\mathrm{L}}(\psi)$ mapping, a standard deviation of $0.5 \mathrm{~cm}$ is assigned to the equilibrium position, i.e. $\sim 2 \%$ of the minor radius. The average profile over the ensemble is taken as the indirect measurement of $u_{\mathrm{p}}$ and one standard deviation its uncertainty 

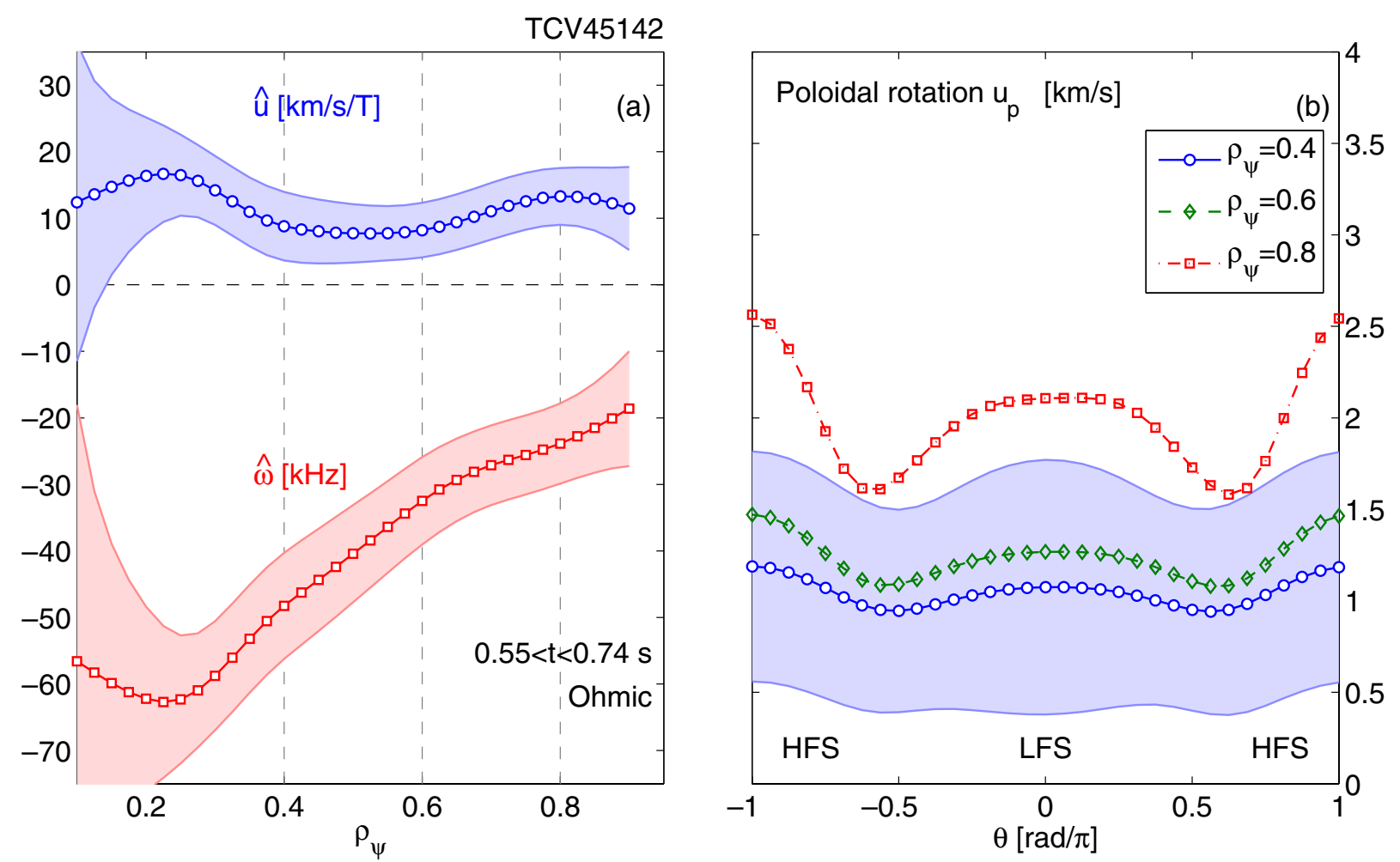

Figure 4. (a) Radial profiles of the flux functions $\hat{\omega}$ and $\hat{u}$, calculated from the toroidal frequency asymmetry, in the ohmic phase of TCV 45142. (b) Dependence of the inferred poloidal rotation on the poloidal angle $\theta$, for three selected flux surfaces $\left(\rho_{\psi}=0.4,0.6,0.8\right)$. Uncertainty corresponding to a $68 \%$ confidence interval is indicated by color bands, for $\rho_{\psi}=0.4$.

TCV45142
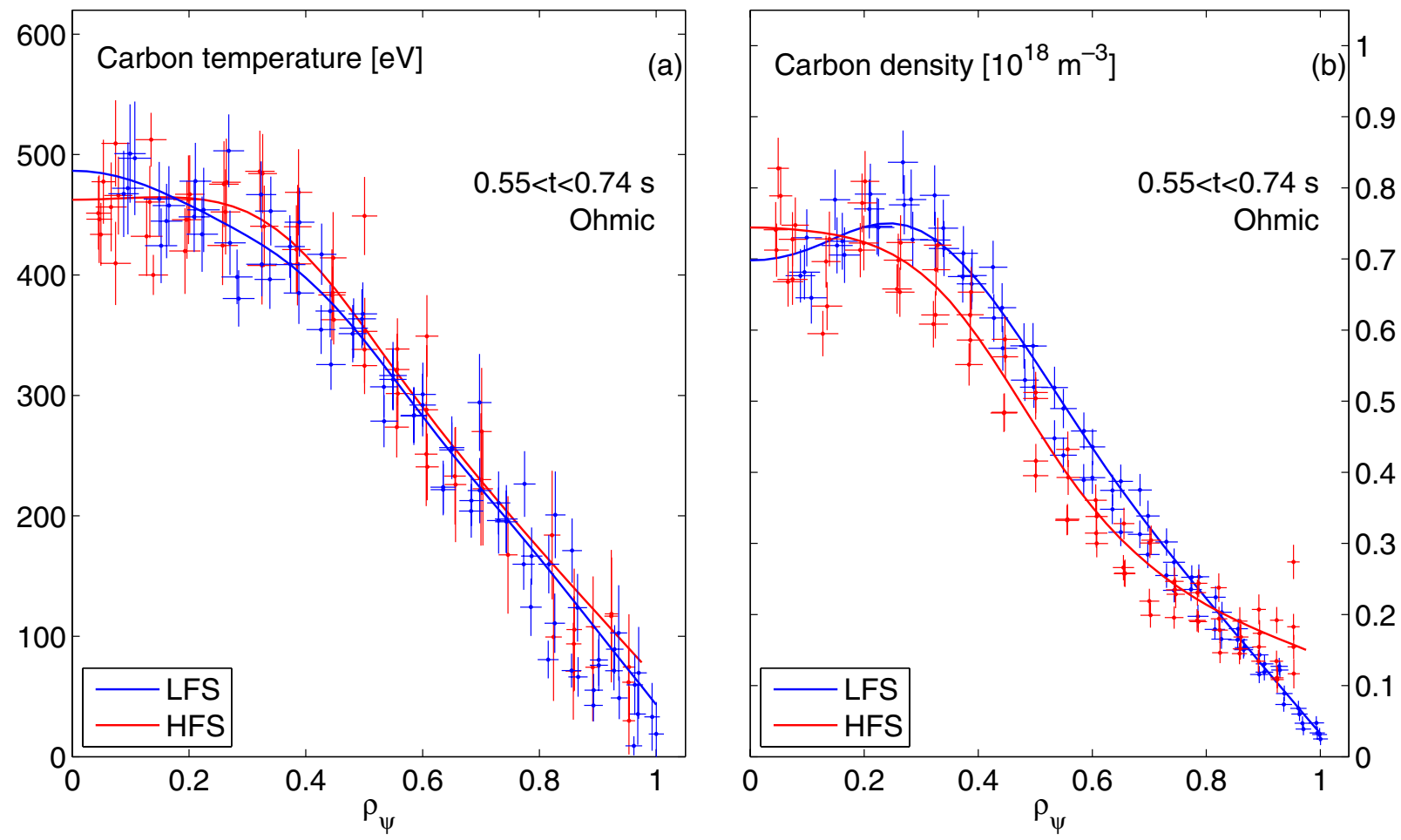

Figure 5. Carbon temperature ( $a$ ) and density $(b)$ during the ohmic phase of TCV discharge 45142. HFS and LHS measurements are presented as functions of the normalized poloidal flux. 

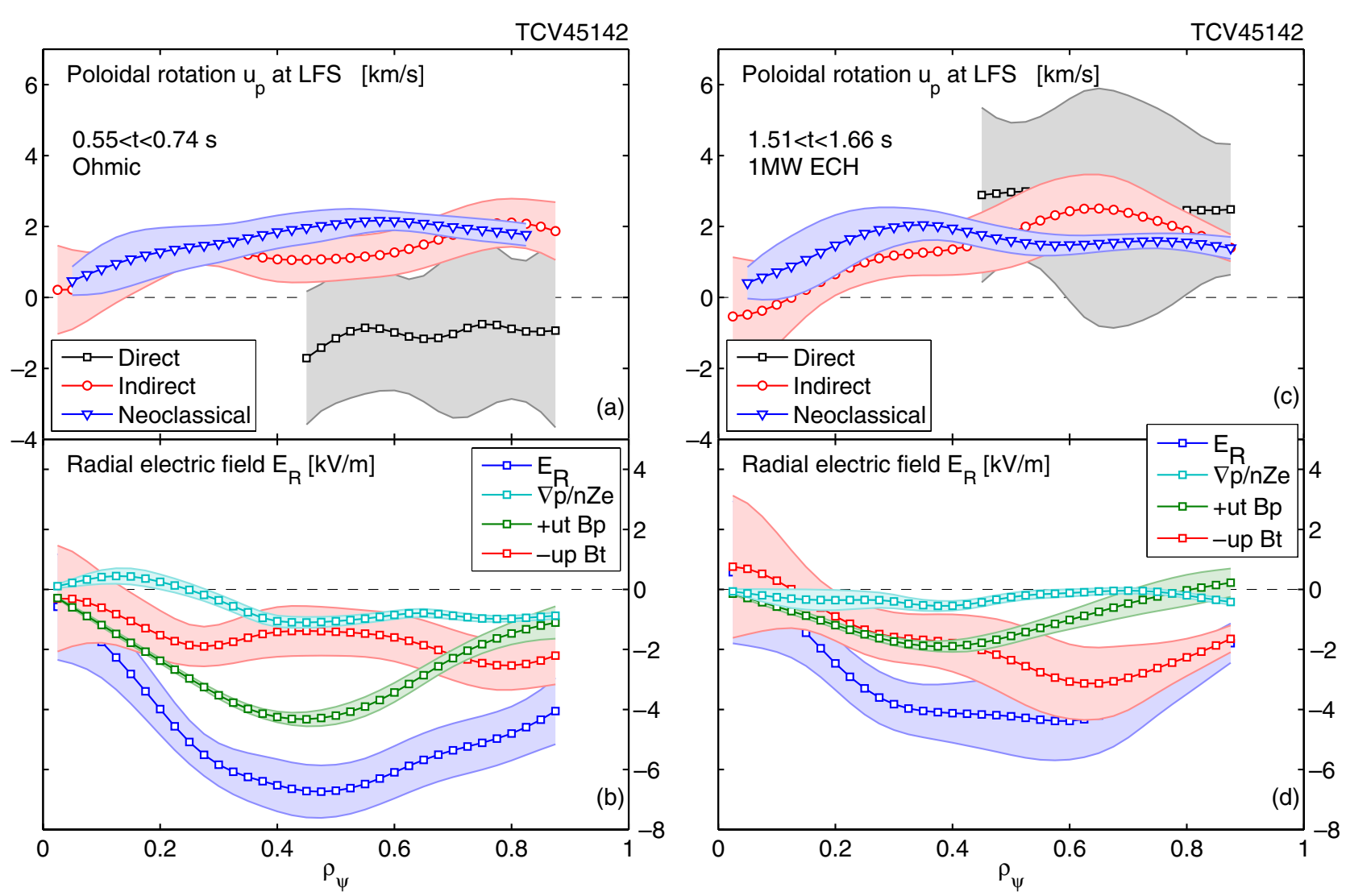

Figure 6. Top panels: comparison between poloidal rotation profiles determined by the indirect measurement, direct measurement and neoclassical prediction. Bottom panels: electric field calculated at the LFS mid-plane, using the force balance equation and the HFS-LFS asymmetry of toroidal frequency.

(i.e. the error bar indicates the conventional $68 \%$ confidence level).

Figure 6 shows the $u_{\mathrm{p}}$ profile evaluated at the outboard mid-plane, for the time windows $0.55-0.74 \mathrm{~s}$ (ohmic) and 1.51-1.66 s (1 MW ECH). The combined uncertainty of $\pm 1 \mathrm{~km} \mathrm{~s}^{-1}$ unequivocally implies that the plasma rotates poloidally in the electron diamagnetic direction (positive values), with velocities of $\approx 2 \mathrm{~km} \mathrm{~s}^{-1}$ at mid-radius, decreasing to zero towards the magnetic axis (where $B_{\mathrm{p}}=0$ ).

TCV's vertical CXRS system provides a direct measurement of $u_{\mathrm{p}}$ at the outboard mid-plane (figure 1). The measured profiles, included in figure 6 , show $u_{\mathrm{p}} \approx 0 \mathrm{~km} \mathrm{~s}^{-1}$ during the ohmic phase, increasing to $\approx 2 \mathrm{kms}-1$ in the $\mathrm{ECH}$ phase. During the ECH phase, improved agreement between the two measurement methods is observed. As the $2 \mathrm{~km} \mathrm{~s}^{-1}$ uncertainty in the direct measurement corresponds to one measurement standard deviation, the direct and indirect measurements may always be considered compatible in the ohmic and ECH phases. It is clear that, for these conditions, given the small value of $u_{\mathrm{p}}$ and the weak $S / N$ and $A / P$ levels, the direct measurement does not attain the accuracy required to unambiguously resolve the poloidal rotation direction.

The bottom panels in figure 6 show the radial electric field calculated from the force balance equation, evaluated at the LFS mid-plane for $\mathrm{C}^{6+}$ impurity using the indirect poloidal rotation measurement. The total $E_{\mathrm{R}}<0$ is of the order of $4-6 \mathrm{kV} \mathrm{m}^{-1}$, with an uncertainty of $\pm 1 \mathrm{kV} \mathrm{m}^{-1}$, unequivocally inwards for most of the plasma cross section.
Note that the contribution from poloidal rotation is substantial, and dominates for the $\mathrm{ECH}$ case. The ability of the indirect measurement to resolve the direction of small poloidal velocities $u_{\mathrm{p}}$ (compared with the direct measurement) allows us to determine the direction of the $E \times B$ drift velocity, a key parameter for the interpretation of microinstabilities studies and fluctuation measurements.

\section{Comparison with neoclassical theory}

The indirect measurement of carbon poloidal rotation has been compared with the neoclassical predictions calculated using the NEOART code [38] in the limit of a stationary plasma composed by deuterium main ions and $\mathrm{C}^{6+}$ impurity. This assumption is supported by the good agreement found between estimates of the effective charge $Z_{\text {eff }}$ obtained assuming carbon dominant impurity with the value estimated from the neoclassical conductivity.

The input density and temperature profiles are taken from Thomson scattering measurements for the electrons and CXRS carbon impurity measurements for the ions. The deuterium density is derived from quasi-neutrality $n_{\mathrm{D}}=n_{\mathrm{e}}-6 n_{\mathrm{C}}$ and the ion species are assumed thermalized $\left(T_{\mathrm{D}}=T_{\mathrm{C}}\right)$. This approach restricts the validity of the calculation to plasma regions where carbon $\mathrm{C}^{6+}$ is the dominant impurity, which corresponds to $\rho_{\psi}<0.8$, for the scenarios addressed in this work. The effect of parallel electric field is included in the calculation, based on the experimental loop voltage. 

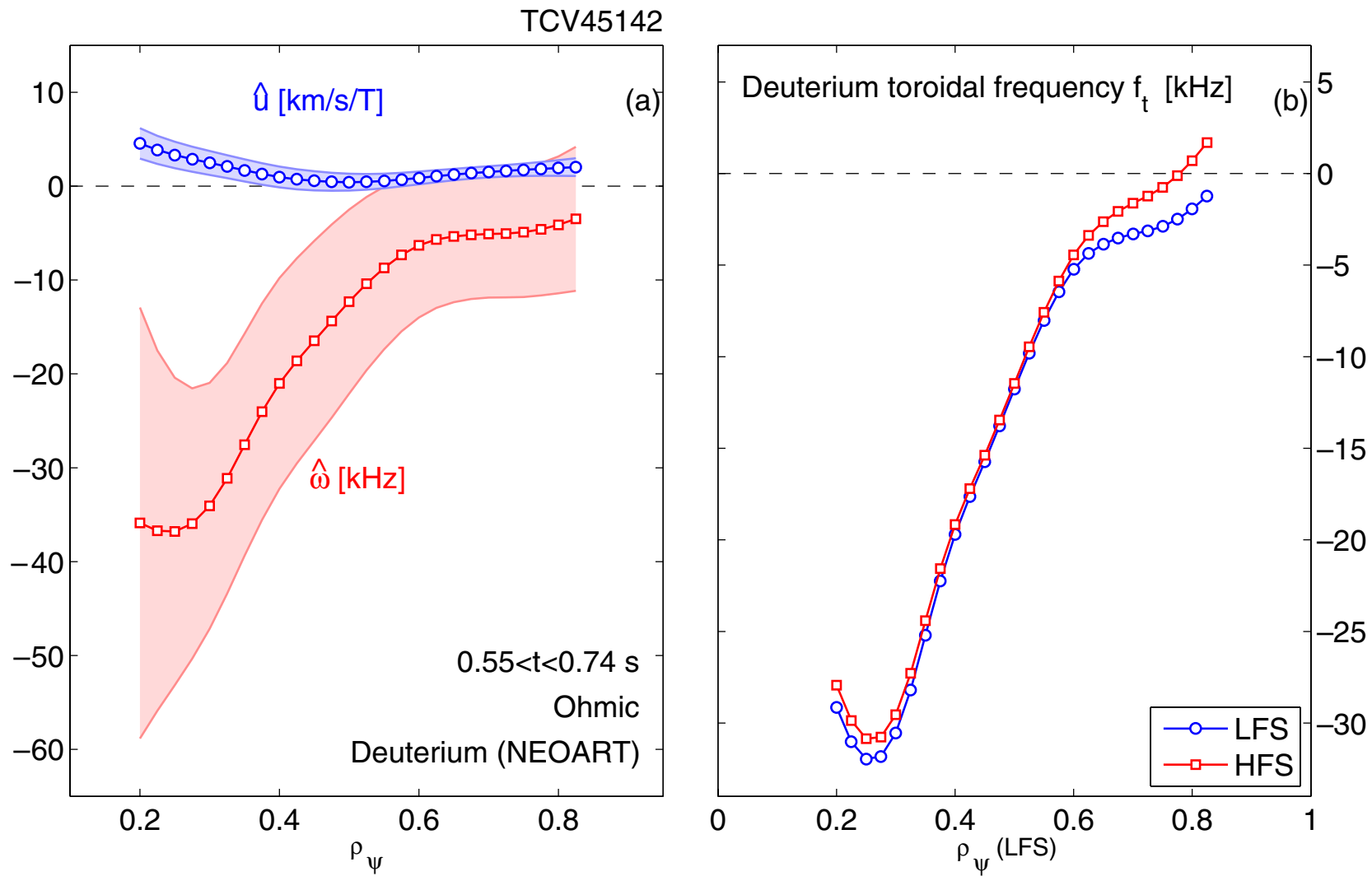

Figure 7. (a) Radial profiles of the flux functions $\hat{\omega}$ and $\hat{u}$, calculated for the main ion species deuterium, using neoclassical predicted poloidal velocity, in the ohmic phase of TCV 45142, and the resulting profiles of toroidal rotation frequency, evaluated at the plasma mid-plane $(b)$.

It is of key importance to assign meaningful uncertainties for the theoretical prediction. This was again accomplished using a Monte Carlo method (see appendix A), that involved performing the NEOART computation for an ensemble of $M=1000$ input datasets, and calculating the ensemble average and standard deviation of each output quantity as the neoclassical prediction and its corresponding uncertainty.

The impact of $H$ fuelling by the DNB was examined by repeating the neoclassical simulations with a small amount of hydrogen in addition to deuterium and $\mathrm{C}^{6+}\left(n_{\mathrm{H}} / n_{\mathrm{D}}=0.15\right)$. The resulting change of the predicted poloidal rotation was found to be negligible and the results shown below ignore hydrogen.

The neoclassical prediction of $\mathrm{C}^{6+}$ poloidal rotation, calculated in the ohmic and EHC phases of TCV 45142, is over plotted in figures $6(a)$ and $(c)$. In both cases, excellent agreement between theory and experiment is found i.e. an electron diamagnetic drift directed $u_{\mathrm{p}}$ of magnitude $1-2 \mathrm{~km} \mathrm{~s}^{-1}$. The calculations also indicate that the higher electron heating does not affect the ion kinetic profiles sufficiently to induce substantial changes in the neoclassical flows.

It should be stressed that since equation (13) applies to each given plasma species separately [29], the impurity species used for the CX measurement (carbon) and main ion species (deuterium) can manifest different degrees of asymmetry. As TCV neoclassical calculations typically yield $\hat{u}_{\mathrm{D}}<\hat{u}_{\mathrm{C}}$, a smaller asymmetry is expected for main ion toroidal rotation and a rigid rotor approximation $\left(u_{\mathrm{t}, \mathrm{D}} \approx \hat{\omega}_{\mathrm{D}} R\right)$ still provides a reasonable approximation of the main ion toroidal plasma flow. To exemplify this, we provide the flow structure for the main ions, for the ohmic phase of TCV 45142, computed using the neoclassical prediction for $\hat{u}_{\mathrm{D}}$ and equation (7) for $\hat{\omega}_{\mathrm{D}}$ (figure 7 ). In this case $\hat{u}_{\mathrm{D}} \lesssim 0.2 \hat{u}_{\mathrm{C}}$ and, as a result, the asymmetry of deuterium toroidal rotation frequency remains everywhere below $3 \mathrm{kHz}$, much smaller than what is observed for carbon.

Exploiting the inboard-outboard asymmetry of $f_{\mathrm{t}}$, we determined the structure of carbon flows and, in particular, the poloidal rotation profile, for a number of plasma discharges with varying magnetic configurations (central column limited or diverted with lower single null), heating scheme (from ohmic up to $1 \mathrm{MW} \mathrm{ECH}$ ) and plasma current and toroidal field direction (independently).

Stationary plasma configurations were obtained at central electron temperature and density of $T_{\mathrm{e}}=800-1200 \mathrm{eV}, n_{\mathrm{e}}=$ $(1.5-6.5) \times 10^{19} \mathrm{~m}^{-3}$. By changing the inductive current, the safety factor, taken at $95 \%$ of the poloidal flux, was varied between $q_{95}=3.5-6.5$. For $\rho_{\psi}<0.8$, the ion collisionality was in the low collisionality banana-plateau regime $0.2<$ $v_{\mathrm{ii}}^{*}<2.4$, with $v_{\mathrm{ii}}^{*}=\left(v_{\mathrm{ii}} q R / \epsilon^{3 / 2}\right) \sqrt{m_{\mathrm{i}} / 2 T_{\mathrm{i}}}$. Depending on the plasma scenario, extremely different (intrinsic) toroidal rotation profiles were observed, including co- and countercurrent directed cases, with values spanning from $u_{\mathrm{t}} \sim-40$ (cnt- $\left.I_{\mathrm{p}}\right)$ to $+20\left(\right.$ co- $\left.I_{\mathrm{p}}\right) \mathrm{km} \mathrm{s}^{-1}$. HFS-LFS $\Delta f_{\mathrm{t}}$ asymmetries of the order of $5-10 \mathrm{kHz}$ were consistently observed. 

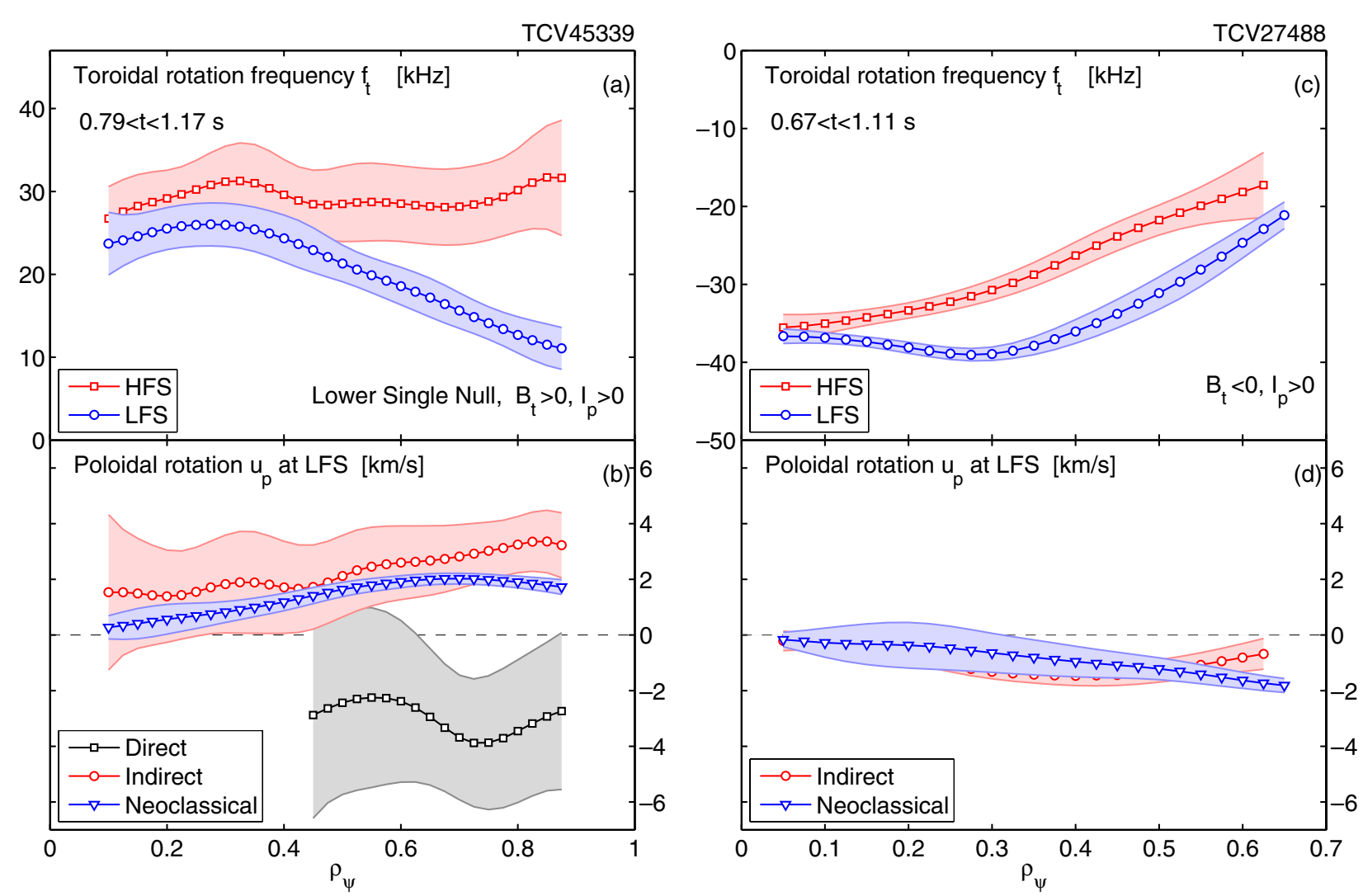

Figure 8. Toroidal rotation profiles $(a),(c)$ and indirect poloidal rotation measurement $(b),(d)$ for two cases: lower single null, co-current rotating discharge (TCV 45339, left) and limited cnt-current rotating with negative $B_{\mathrm{t}}$ and $I_{\mathrm{p}}$ (TCV 27098, right). For the second discharge the HFS CXRS measurement coverage was limited to $0<\rho_{\psi}<0.6$.

Figure 8 details the results obtained for two cases. In TCV discharge $45339\left(I_{\mathrm{p}}=+250 \mathrm{kA}, B_{\mathrm{t}}=+1.42 \mathrm{~T} n_{\mathrm{e}}=\right.$ $4 \times 10^{19} \mathrm{~m}^{-3}$ ) plasma was confined in diverted lower single null configuration. As described in previous publications, for these conditions, a stationary co-current directed $u_{\mathrm{t}}$ profile develops [36], here reaching values $25 \mathrm{~km} \mathrm{~s}^{-1}$ at the magnetic axis. A significant asymmetry of $f_{\mathrm{t}}$ is observed for $\rho_{\psi}>0.4$, which is outside the sawtooth inversion radius, increasing towards the plasma boundary to up to $20 \mathrm{kHz}$. The associated $u_{\mathrm{p}}$ of $2 \mathrm{~km} \mathrm{~s}^{-1}$ for $0.5<\rho_{\psi}<0.9$ agrees with the neoclassical prediction. Note that the direct measurement does not provide the correct sign for $u_{\mathrm{p}}$, albeit large direct statistical uncertainties of the order of $3 \mathrm{~km} \mathrm{~s}^{-1}$ do not permit further comparison with the indirect measurement. However, the indirect measurements favourably compare with the neoclassical predictions.

TCV discharge 27488 has a limited plasma configuration with plasma parameters close to that of TCV 45142 shown in figure 3 , but with reversed toroidal magnetic field direction $\left(I_{\mathrm{p}}=+160 \mathrm{kA}, B_{\mathrm{t}}=-1.42 \mathrm{~T} n_{\mathrm{e}}=4 \times 10^{19} \mathrm{~m}^{-3}\right)$. In this experiment, only a preliminary arrangement of the toroidal HFS view was available [31], with nine channels covering the inboard mid-plane region from the magnetic axis to $\rho_{\psi} \approx 0.6$. With active to passive signal ratios of $\approx 50 \%$ significantly better accuracy in the $u_{\mathrm{t}}$ measurement was attained. The toroidal frequency asymmetry develops at inner location, with $f_{\mathrm{t}, \mathrm{H}}-f_{\mathrm{t}, \mathrm{L}}>0$, i.e. in the co-current direction, as found for the $B_{\mathrm{t}}>0$ case. This indicates that the flux function $\hat{u}(\psi)$ changes sign upon $B_{\mathrm{t}}$ reversal. Consequently, the inferred $u_{\mathrm{p}}$ also changes sign, with $u_{\mathrm{p}} \approx-1 \mathrm{~km} \mathrm{~s}^{-1}$ inferred at mid-radius. Poloidal rotation remains directed in the electron diamagnetic drift, in agreement with the neoclassical predictions.

The results obtained over the entire dataset are summarized in figure 9 , where the indirect measurements of $u_{\mathrm{p}}$ are plotted as a function of the neoclassical predictions. Here, $u_{\mathrm{p}}$ is evaluated at outboard mid-plane and data are averaged over radial intervals of $\Delta \rho_{\psi}=0.1$ from $\rho_{\psi}=0.2$ to $\rho_{\psi}=$ 0.8 . Within an accuracy of $1 \mathrm{~km} \mathrm{~s}^{-1}$, neoclassical prediction and experimental measurement correlate well. Inclusion of data from negative $B_{\mathrm{t}}$ experiments populates the graph with negative values. Data-points marked with black faces correspond to measurements with central $u_{\mathrm{t}}$ in the co-current direction. Within the error bar, $u_{\mathrm{p}}$ was always measured and predicted in the electron diamagnetic drift direction.

Strictly speaking, since NEOART assumes the flow structure of equation (12), a comparison between experimental observations and modelling is meaningful only if flows can be expressed in this form, with equation (16) used to infer $\hat{u}$. In a few cases, a sizeable difference between HFS and LFS carbon density was measured, with $n_{\mathrm{C}, \mathrm{H}} / n_{\mathrm{C}, \mathrm{L}} \approx 0.7$ at mid-radius. These observations of poloidal asymmetry of the carbon density remain, to date, unexplained and will be the subject of future investigation. Their impact on the indirect poloidal rotation measurement was estimated using equation (18) instead of equation (16) to determine $u_{\mathrm{p}}$. At the LFS mid-plane, the poloidal rotation inferred using equation (18) is systematically lower by about $0.5 \mathrm{~km} \mathrm{~s}^{-1}$. 


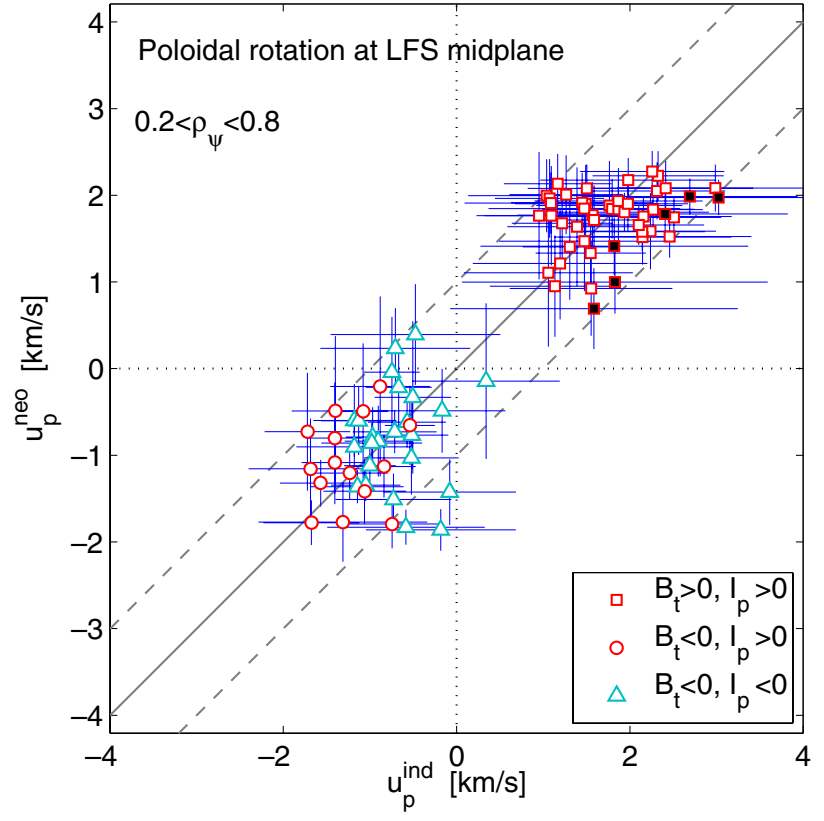

Figure 9. Indirect measurement of poloidal rotation at LFS mid-plane as a function of the neoclassical prediction, for a range of L-mode discharges. Measurements at radial locations $0.2<\rho_{\psi}<0.8$, separated by $\Delta \rho_{\psi}=0.1$, are shown. Data points marked in black correspond to plasmas with central $u_{\mathrm{t}}$ directed in the direction of plasma current.

\section{Discussion and conclusions}

The reported investigations demonstrate that, by exploiting the poloidal asymmetry of toroidal rotation, the complete bi-dimensional plasma flow can be obtained, which includes an accurate indirect measurement of poloidal rotation relevant for a comparison with theoretical models.

The major advantage of this technique is that any poloidal velocity is amplified in the difference of toroidal frequency $\Delta f_{\mathrm{t}}$ by a factor $\approx 4 q / R_{0}$. In fact, Doppler shifts associated with the measurement of toroidal rotation generally exceed the spectroscopic measurement uncertainties, that often dominate the direct measurement of $\mathrm{km} \mathrm{s}^{-1}$ plasma poloidal velocity. A measurement of the difference between two Doppler shifts is also less affected by uncertainties on the calibration wavelength of the spectroscopic line. Although not yet implemented on TCV, this could be further improved by using the same spectrometer to measure both inboard and outboard values where only the stability of the spectrometer's dispersion would be pertinent. For an active CX measurement, this indirect measurement is less vulnerable to issues associated with the finite lifetime of the excited state of the emitting impurity $[12,39]$ that influence observational views perpendicular to the magnetic field.

On the other hand, uncertainties related to the mapping of HFS and LFS profiles on a flux variable and increased error associated with a difference of two measured quantities can limit the precision of the measurement and, for CX measurements, obtaining a significant neutral density on the HFS with LFS injection is probably not viable in large machines.

In the analyses performed for this paper, which mostly used existing data, the $\mathrm{CX}$ diagnostic did not benefit from optimal $S / N$ and/or active to passive signal ratios. Nevertheless, the indirect measurement was successful in determining a poloidal rotation direction with a high degree of confidence that equalled and in most cases outperformed the direct measurement.

The indirect measurement method was applied to L-mode tokamak plasmas but can be employed for any toroidal axisymmetrical confinement configuration, provided that flows are divergence free on flux surfaces. This requires the component of the flow perpendicular to the flux surface to be zero to leading order. In the presence of strong particle sources or large variation in radial particle transport this condition may not be satisfied: here the flows cannot be expressed in the form of equation (12) and asymmetries can arise independently of the poloidal rotation value $[13,30]$. The presence of non-axisymmetric magnetic perturbations, such as those associated with MHD modes or externally applied 3D fields, could also, in principle, reduce the reliability of this approach.

Although the indirect measurement was tested for a charge exchange diagnostic, it is applicable for any velocity measurement technique. For example, in ITER [40], an appropriate arrangement of a x-ray crystal based diagnostic [41] could provide a viable way to measure $u_{\mathrm{t}}, u_{\mathrm{p}}$ and $E_{r}$ in the plasma core, where $\mathrm{CX}$ diagnostics will be limited by beam attenuation.

For the dataset presented, in the region of the plasma where the indirect measurement is thought reliable $\left(\rho_{\psi}<\right.$ 0.8 ), the poloidal rotation of the carbon $\mathrm{C}^{6+}$ impurity was found consistently in the electron diamagnetic drift direction and in quantitative agreement with neoclassical predictions. Although the parameter space explored was large, without auxiliary ion heating, only a relatively small range of $\left|u_{\mathrm{p}}\right|<$ $2 \mathrm{~km} \mathrm{~s}^{-1}$ was sampled, limiting the capability of investigating the dependence of the measured poloidal rotation on plasma parameters. In particular, no clear correlation emerged with the gradients of ion temperature or carbon density $\left(\mathrm{d} T_{\mathrm{i}} / \mathrm{d} R=\right.$ $0-3.5 \mathrm{kev} \mathrm{m}^{-1}, \quad \mathrm{~d} n_{\mathrm{C}} / \mathrm{d} R=\left(0.1-2 \times 10^{19} \mathrm{~m}^{-3} \mathrm{~m}^{-1}\right)$. Furthermore, for the dataset included in the study, the ion collisionality remained in the low collisionality banana-plateau regime $0.2<v_{\mathrm{ii}}^{*}<2.4$, with $v_{\mathrm{ii}}^{*}=\left(v_{\mathrm{ii}} q R / \epsilon^{3 / 2}\right) \sqrt{m_{\mathrm{i}} / 2 T_{\mathrm{i}}}$. More stringent tests of the theoretical predictions could be performed by operating at reduced toroidal field.

Although no deviation from neoclassical poloidal rotation emerged from the reported TCV dataset, such conditions may exist for other plasma scenarios.

\section{Appendix A. Monte Carlo approach to the evaluation of uncertainties}

Even where all experimental quantities are known with appropriate uncertainties, error propagation for derived quantities is often complex. This is the case, for example, when gradients of quantities resulting from fitting procedures are concerned, or when the derived quantity is the result of complex numerical computation. In these and other cases, a practical solution is to adopt a Monte Carlo approach.

Consider a function of multiple variables $f(x, y, z \ldots)$, where $x, y, z$ correspond to quantities that are measured experimentally. Although not necessary, in this context, it may be helpful to think $x$ as a space variable (e.g. $\rho_{\psi}$ ) and $y$, 
$z$, etc as extensive quantities evaluated at the $x$ locations (e.g. $T_{\mathrm{e}}$ ). The function $f$ can contain an explicit dependence on the gradients of the variables. For example it may represent a gradient operator or a complex computation involving nonlinear operations, such as a non-linear fitting optimization.

For each variable, for instance $x$, the experimental measurements are represented by a set of $N$ values $\left\{x_{i}\right\}_{i=1, \ldots, N}$, with their uncertainties $\left\{\delta x_{i}\right\}$. We assume that the experimental quantities obey a normal distribution, and that the uncertainties represent a statistical variance. Our scope is to obtain a set $\left\{f_{i}, \delta f_{i}\right\}$. For each measurement $x_{i}$ a set of $M$ 'virtual' measurements $\left\{x_{i j}\right\}_{j=1, \ldots, M}$ is generated randomly, following a normal distribution, in such a way that:

$$
\begin{aligned}
x_{i} & =\frac{1}{M} \sum_{j} x_{i j}, \\
\delta x_{i}^{2} & =\frac{1}{M} \sum_{j}\left(x_{i j}-x_{i}\right)^{2} .
\end{aligned}
$$

The same expansion is performed for all variables. For each index $j=1, \ldots, M$ a particular evaluation of the $f$ function is computed:

$$
f_{i j}=f\left(x_{i j}, y_{i j}, z_{i j}\right) \text {. }
$$

The final result is obtained taking the average and standard deviation over the ensemble $\left\{f_{i j}\right\}_{j=1, \ldots, M}$ :

$$
\begin{aligned}
f_{i} & =\frac{1}{M} \sum_{k} f_{i j}, \\
\delta f_{i}^{2} & =\frac{1}{M} \sum_{j}\left(f_{i j}-f_{i}\right)^{2} .
\end{aligned}
$$

Note that the final uncertainty conserves the statistical meaning of variance.

The advantage of this approach is that it does not require analytic error propagation formulae that in many cases are difficult to derive and it does not use a linear expansion typically favoured in error propagation analysis.

In the context of this paper, the method has been applied for the fitting of the experimental profiles; for the calculation of the $\hat{\omega}(\psi)$ and $\hat{u}(\psi)$; and in the calculation of the neoclassical quantities.

\section{Appendix B. Formulation independent of coordinate conventions}

In section 2, the local poloidal and toroidal rotation have been related to the two flux surface functions $\hat{\omega}$ and $\hat{u}$. The latter can take different signs, and the respective contributions to the total flow or to the radial electric field might cancel out in some cases. Therefore, it is important to have the correct signs when defining a poloidal or toroidal component.

In practice, the task is often complicated by the use of many different conventions for the direction of increasing toroidal and poloidal angles, the sign and normalization of the poloidal flux, etc. The tokamak coordinate convention scheme (COCOS) proposed in [25] represents a powerful tool to account explicitly for the sign conventions.

In this section, we present the formulae most relevant to the indirect measurement of poloidal rotation, expressed in the COCOS formalism. These expressions contain the information about the coordinate conventions in a parametrized form. Once a convention system is specified, the corresponding formulae are readily obtained.

In the COCOS formalism the magnetic field vector takes the form

$$
\boldsymbol{B}=\tilde{F} \nabla \varphi+\sigma_{B \mathrm{p}} \frac{1}{(2 \pi)^{e_{B \mathrm{p}}}} \nabla \varphi \times \nabla \psi
$$

where $\tilde{F}=\operatorname{sign}(B \cdot \nabla \varphi) F$, with $F=\left|R B_{\mathrm{t}}\right|>0$. The values of the parameters $\sigma_{B \mathrm{p}}$ and $e_{B \mathrm{p}}$ are uniquely defined by the COCOS index associated with the conventions (table I of [25]). For example, the conventions used throughout this work correspond to the COCOS index 2, for which $\sigma_{B \mathrm{p}}=1$ and $e_{B \mathrm{p}}=0$.

The general expressions, independent of coordinate conventions, for the flux functions $\hat{\omega}$ and $\hat{u}$ are

$\hat{\omega}(\psi)=(2 \pi)^{e_{B \mathrm{p}}} \sigma_{B \mathrm{p}}\left(\frac{\partial \phi}{\partial \psi}+\frac{1}{Z e n} \frac{\partial p}{\partial \psi}\right)$,

$\hat{u}(\psi)=\frac{u_{\|}}{B}-(2 \pi)^{e_{B \mathrm{p}}} \sigma_{B \mathrm{p}}\left(\frac{\partial \phi}{\partial \psi}+\frac{1}{Z e n} \frac{\partial p}{\partial \psi}\right) \frac{\tilde{F}}{B^{2}}$.

The flux functions $\hat{\omega}$ and $\hat{u}$ combine to give the toroidal flow of the given species:

$$
u_{\varphi}=\hat{\omega}(\psi) R+\hat{u}(\psi) \tilde{F} / R
$$

The latter equation, evaluated at the inboard and outboard location of a flux surface, is then used to obtain the flux functions from the toroidal flow measurement:

$\hat{\omega}(\psi)=\left(u_{\mathrm{t}, \mathrm{H}} R_{\mathrm{H}}-u_{\mathrm{t}, \mathrm{L}} R_{\mathrm{L}}\right) \times\left(R_{\mathrm{H}}^{2}-R_{\mathrm{L}}^{2}\right)^{-1}$,

$\hat{u}(\psi)=\frac{1}{\tilde{F}}\left(\frac{u_{\mathrm{t}, \mathrm{H}}}{R_{\mathrm{H}}}-\frac{u_{\mathrm{t}, \mathrm{L}}}{R_{\mathrm{L}}}\right) \times\left(\frac{1}{R_{\mathrm{H}}^{2}}-\frac{1}{R_{\mathrm{L}}^{2}}\right)^{-1}$.

Following the COCOS system, the component of the electric field perpendicular to the flux surface reads

$$
\frac{\boldsymbol{E} \cdot \nabla \psi}{|\nabla \psi|}=\left[\frac{1}{Z e n} \frac{\partial p}{\partial \psi}-\hat{\omega}(\psi) \frac{\sigma_{B \mathrm{p}}}{(2 \pi)^{e_{B \mathrm{p}}}}\right](2 \pi)^{e_{B \mathrm{p}}} R B_{\mathrm{p}},
$$

where $B_{\mathrm{p}}=|\nabla \varphi \times \nabla \psi|=|\nabla \psi| /\left[(2 \pi)^{e_{B_{\mathrm{p}}}} R\right]$ is the norm of the poloidal magnetic field. It is important to note that, depending on the conventions and the direction of the plasma current, $\operatorname{sign}(\nabla \psi)$ can be positive or negative. In order to have the electric field related to an outward radial direction, one needs to use the same transformation as in equation (24) of [25].

\section{Acknowledgments}

This work was supported in part by the Swiss National Science Foundation, US Department of Energy (Contract Nos DEFG02-06ER54867, DE-AC02-09CH11466) and partly funded by EURATOM under EFDA baseline and priority support No WP11-TRA-04-01-03/CEA.

\section{References}

[1] Betti R. and Freidberg J.P. 2000 Radial discontinuities in tokamak magnetohydrodynamic equilibria with poloidal flow Phys. Plasmas 7 2439-48

[2] Guazzotto L., Betti R., Manickam J. and Kaye S. 2004 Numerical study of tokamak equilibria with arbitrary flow Phys. Plasmas 11 604-14 
[3] Kissick M.W., Leboeuf J.-N., Cowley S.C. and Dawson J.M. 2001 Poloidal rotation effects on a simulated resistive kink mode Phys. Plasmas 8 174-9

[4] Sato M., Hamaguchi S. and Wakatani M. 2003 Destabilization of nonlinear resistive wall mode due to suppression of poloidal rotation in a cylindrical tokamak Phys. Plasmas 10 187-94

[5] Aiba N., Shiraishi J. and Tokuda S. 2011 Impact of plasma poloidal rotation on resistive wall mode instability in toroidally rotating plasmas Phys. Plasmas 18022503

[6] Aiba N., Furukawa M., Hirota M., Oyama N., Kojima A., Tokuda S. and Yagi M. 2011 Mechanisms of plasma rotation effects on the stability of type-I edge-localized mode in tokamaks Nucl. Fusion 51073012

[7] Dif-Pradalier G., Grandgirard V., Sarazin Y., Garbet X. and Ghendrih Ph. 2009 Interplay between gyrokinetic turbulence, flows, and collisions: perspectives on transport and poloidal rotation Phys. Rev. Lett. 103065002

[8] Bell R.E., Andre R., Kaye S.M., Kolesnikov R.A. LeBlanc B.P., Rewoldt G., Wang W.X. and Sabbagh S.A. 2010 Comparison of poloidal velocity measurements to neoclassical theory on the national spherical torus experiment Phys. Plasmas 17082507

[9] Bell R.E. and Feder R. 2010 Measurement of poloidal velocity on the national spherical torus experiment (invited) Rev. Sci. Instrum. 81 10D724

[10] Isler R.C. 1994 An overview of charge-exchange spectroscopy as a plasma diagnostic Plasma Phys. Control. Fusion 36 171-208

[11] Thomas D.M. 2012 Beams, brightness, and background: using active spectroscopy techniques for precision measurements in fusion plasma research Phys. Plasmas 19056118

[12] Solomon W.M., Burrell K.H., Gohil P., Groebner R.J. and Baylor L.R. 2004 Extraction of poloidal velocity from charge exchange recombination spectroscopy measurements Rev. Sci. Instrum. 75 3481-6

[13] Marr K.D., Lipschultz B., Catto P.J., McDermott R.M., Reinke M.L. and Simakov A.N. 2010 Comparison of neoclassical predictions with measured flows and evaluation of a poloidal impurity density asymmetry Plasma Phys. Control. Fusion 52055010

[14] Viezzer E. et al and the ASDEX Upgrade Team 2012 Investigations on the edge radial electric field at asdex upgrade Proc. 39th EPS Conf. on Plasma Physics (Stockholm, Sweden, 2-6 July 2012) (ECA) vol 36F ed S. Ratynskaya et al (European Physical Society) O5.118 http://ocs.ciemat.es/epsicpp2012pap/html/index.html

[15] Field A.R., McCone J., Conway N.J., Dunstan M., Newton S. and Wisse M. 2009 Comparison of measured poloidal rotation in mast spherical tokamak plasmas with neo-classical predictions Plasma Phys. Control. Fusion 51105002

[16] Meigs A.G. and Rowan W.L. 1994 Impurity poloidal rotation velocity in tokamaks Phys. Plasmas $1960-7$

[17] Kim J., Burrell K.H., Gohil P., Groebner R.J., Kim Y.-B., John H.E.St., Seraydarian R.P. and Wade M.R. 1994 Rotation characteristics of main ions and impurity ions in H-mode tokamak plasma Phys. Rev. Lett. 72 2199-202

[18] Solomon W.M., Burrell K.H., Andre R., Baylor L.R., Budny R., Gohil P., Groebner R.J., Holcomb C.T., Houlberg W.A. and Wade M.R. 2006 Experimental test of the neoclassical theory of impurity poloidal rotation in tokamaks Phys. Plasmas 13056116

[19] Crombé K. et al 2005 Poloidal rotation dynamics, radial electric field, and neoclassical theory in the JET internal-transport-barrier region Phys. Rev. Lett. 95155003

[20] Fenzi C. et al and the Tore Supra Team 2011 On plasma rotation with toroidal magnetic field ripple and no external momentum input Nucl. Fusion 51103038

[21] Chrystal C., Burrell K.H., Grierson B.A., Groebner R.J. and Kaplan D.H. 2012 Calculation of impurity poloidal rotation from measured poloidal asymmetries in the toroidal rotation of a tokamak plasma Rev. Sci. Instrum. 83 10D501

[22] Hofmann F. et al 1994 Creation and control of variably shaped plasmas in TCV Plasma Phys. Control. Fusion 36 B277-87

[23] Hirschman S.P. and Sigmar D.J. 1981 Neocalssical transport of impurities in tokamak plasmas Nucl. Fusion 21 1079-201

[24] Helander P. and D.J. Sigmar 2002 Collisional Transport in Magnetized Plasmas (Cambridge Monographs on Plasma Physics) (Cambridge: Cambridge University Press)

[25] Sauter O. and Medvedev S.Yu. 2013 Tokamak coordinate conventions: Cocos Comput. Phys. Commun. 184293

[26] Hinton F.L. and Wong S.K. 1985 Neoclassical ion transport in rotating axisymmetric plasmas Phys. Fluids 28 3082-98

[27] Hsu C.T. and Sigmar D.J. 1990 Transport induced by ion-impurity friction in strongly rotating, collisional tokamak plasma Plasma Phys. Control. Fusion 32499

[28] Fülöp T. and Helander P. 2001 Nonlinear neoclassical transport in toroidal edge plasmas Phys. Plasmas 8 3305-13

[29] Kim Y.B., Diamond P.H. and Groebner R.J. 1991 Neoclassical poloidal and toroidal rotation in tokamaks Phys. Fluids B 3 2050-60

[30] Pütterich T., Viezzer E., Dux R., McDermott R.M. and the ASDEX Upgrade Team 2012 Poloidal asymmetry of parallel rotation measured in asdex upgrade $\mathrm{Nucl}$. Fusion 52083013

[31] Bortolon A. 2009 Plasma rotation and momentum transport studies in the TCV tokamak based on charge exchange spectroscopy measurements $P h D$ Thesis cole Politechnique Fédérale de Lausanne

[32] Duval B.P., Bortolon A., Karpushov A., Pitts R.A., Pochelon A., Scarabosio A. and the TCV Team 2007 Bulk plasma rotation in the TCV tokamak in the absence of external momentum input Plasma Phys. Control. Fusion 49 B195-209

[33] Karpushov A.N. 2012 Charge exchange recombination spectroscopy measurement of ion temperature, rotation and impurity density profiles on the TCV tokamak Proc. 39th European Physical Society Conf. on Plasma Physics (Stockholm, Sweden, 2-6 July 2012) (ECA) vol 36F ed S. Ratynskaya et al (European Physical Society) P2.037 (contributed poster) http://ocs.ciemat.es/epsicpp2012pap/ html/index.html

[34] Karpushov A.N., Andrebe Y., Duval B.P. and Bortolon A. 2009 Proc. 25th Symp. on Fusion Technology (Rostock, Germany, 15-19 September 2008); The diagnostic neutral beam injector with arc-discharge plasma source on the TCV tokamak Fusion Eng. Des. 84 993-7

[35] Bortolon A., Duval B.P., Pochelon A. and Scarabosio A. 2006 Observation of spontaneous toroidal rotation inversion in ohmically heated tokamak plasmas Phys. Rev. Lett. 97235003

[36] Duval B.P., Bortolon A., Karpushov A., Pitts R.A., Pochelon A., Sauter O., Scarabosio A., Turri G. and the TCV Team 2008 Spontaneous L-mode plasma rotation scaling in the TCV tokamak Phys. Plasmas 15056113

[37] Scarabosio A., Bortolon A., Duval B.P., Karpushov A. and Pochelon A. 2006 Toroidal plasma rotation in the TCV tokamak Plasma Phys. Control. Fusion 48 663-83

[38] Peeters A.G. 2000 Reduced charge state equations that describe Pfirsch Schlüter impurity transport in tokamak plasma Phys. Plasmas 7 268-75

[39] Bell R.E. and Synakowski E.J. 2000 New understanding of poloidal rotation measurements in a tokamak plasma Atomic Processes in Plasmas: 12th Topical Conf.; AIP Conf. Proc. 547 39-52

[40] ITER Physics Basis Editors et al 1999 Nucl. Fusion 39 2137-638

[41] Hill K.W. et al 2008 A spatially resolving X-ray crystal spectrometer for measurement of ion-temperature and rotation-velocity profiles on the alcator c-mod tokamak Rev. Sci. Instrum. 79 10E320 